\title{
Core Equivalence in Economy for Modal Logic*
}

\author{
Takashi Matsuhisa ${ }^{\star \star}$ \\ Department of Liberal Arts and Sciences, Ibaraki National College of Technology \\ Nakane 866, Hitachinaka-shi, Ibaraki 312-8508, Japan. \\ mathisa@ge.ibaraki-ct.ac.jp
}

\begin{abstract}
We investigate a pure exchange economy under uncertainty with emphasis on the logical point of view; the traders are assumed to have a multi-modal logic with non-partitional information structures. We propose a generalized notion of rational expectations equilibrium for the economy and we show the core equivalence theorem: The ex-post core for the economy coincides with the set of all its rational expectations equilibria.
\end{abstract}

Keywords: Multi-modal logic, Pure exchange economy under reflexive information structure, Ex-post core, Rational expectations equilibrium, Core equivalence theorem.

Journal of Economic Literature Classification: D51, D84, D52, C72.

\section{Introduction}

This article relates economies and multi-agent modal logic. Let us consider a pure exchange atomless economy under uncertainty. As far as the standard notion of economy either with complete information or with incomplete information, the role of traders' knowledge and beliefs remains obscured: The economy has not been investigated from the epistemic point of view. Here this article aims to fill that gap. Specifically, we seek epistemic conditions of traders' knowledge for the equivalence between equilibria and core in a pure exchange atomless economy under uncertainty.

The purposes of this article are: First to propose the multi-modal logic KT by which the traders use making their decision, secondly to establish the extended notion of rational expectations equilibrium for the economy, and finally to investigate the relationship between the ex-post core and the rational expectations equilibrium allocations with emphasis on modal logical point of view.

The stage is set by the following: Suppose that the trader have the multiagent modal logic $\mathbf{K T}$ : It is an extension of the propositional logic with many modal operators requiring only the axiom $(\mathrm{T})$ " each traders does not know a sentence whenever it is not true." The logic have non-partitional information structures, each of which gives an interpretation of the logic. Each trader has

* This is an extended abstract and the final form will be published elsewhere.

** Partially supported by the Grant-in-Aid for Scientific Research(C)(2)(No.14540145) in the Japan Society for the Promotion of Sciences. 
own utility function which is measurable, but he/she is not assumed to know the function completely. It is shown that

Main Theorem. (Core equivalence theorem). In a pure exchange atomless economy under generalized information, assume that the traders have the multi-modal logic KT and they are risk averse. Then the ex-post core coincides with the set of all rational expectations equilibrium allocations for the economy.

Many authors have investigated several notions of core in an economy under asymmetric information (e.g., Wilson [11], Volij [10], Einy et al [5] and others). The serious limitations of the analysis in these researches are its use of the 'partition' structure by which the traders receive information. The structure is the Kripke semantics for the modal logic $\mathbf{S 5} ;{ }^{1}$ it is obtained if each trader $t$ 's possibility operator $P_{t}: \Omega \rightarrow 2^{\Omega}$ assigning to each state $\omega$ in a state space $\Omega$ the information set $P_{t}(\omega)$ that $t$ possesses in $\omega$ is reflexive, transitive and symmetric. From the epistemic point of view, this entails $t$ 's knowledge operator $K_{t}: 2^{\Omega} \rightarrow 2^{\Omega}$ that satisfies 'Truth' axiom $\mathbf{T}: K_{t}(E) \subseteq E$ (what is known is true), the 'positive introspection' axiom 4: $K_{t}(E) \subseteq K_{t}\left(K_{t}(E)\right.$ ) (we know what we do) and the 'negative introspection' axiom 5: $\bar{\Omega} \backslash K_{t}(E) \subseteq K_{t}\left(\Omega \backslash K_{t}(E)\right)$ (we know what we do not know). ${ }^{2}$

One of these requirements, symmetry (or the equivalent axiom 5), is indeed so strong that describes the hyper-rationality of traders, and thus it is particularly objectionable. The recent idea of 'bounded rationality' suggests dropping such assumption since real people are not complete reasoners. In this article we weaken both transitivity and symmetry imposing only reflexivity. As has already been pointed out in the literature, this relaxation can potentially yield important results in a world with imperfectly Bayesian agents (e.g. Geanakoplos [7]).

The idea has been performed in different settings. Among other things Geanakoplos [7] showed the no speculation theorem in the extended rational expectations equilibrium under the assumption that the information structure is reflexive, transitive and nested (Corollary 3.2 in Geanakoplos [7]). The condition 'nestedness' is interpreted as a requisite on the 'memory' of the trader.

However all those researches have been lacked the logics that represents the traders' knowledge. This article proposes the multi-modal logic of the traders and the economies under generalized information structure as the models for the logic. In the structure we shall relax the transitivity, and we extend the ex-post core equivalence theorem of Einy et al [5] into the models with removing out transitivity and symmetry.

This article is organized as follows: In Section 2 we present the multi-modal logic KT and give its finite model property. Further we introduce the notion "economy for logic KT", a generalized notion of rational expectations equilibrium and ex-post core for the economy. Section 3 gives the existence theorem of rational expectations equilibrium. In Section 4 we give an outline of the proof of

\footnotetext{
$\overline{{ }^{1} \text { C.f.: Chellas }}[3]$, Fagin, Halpern et al [6].

${ }^{2}$ C.f.: Bacharach [2], Fagin, Halpern et al [6].
} 
Main theorem. Finally we conclude by giving some remarks about the assumptions of the theorem. The detailed proofs except for Theorem 1 and discussions are given in Matsuhisa, Ishikawa and Hoshino [9].

\section{Economy for Multi-modal Logic}

\subsection{Logic of Knowledge KT}

Let $T$ be a set of traders and $t \in T$. Let us modal logics for traders as folows: The sentences of the language form the least set containing each atomic sentence $\mathbf{P}_{m}(m=0,1,2, \ldots)$ closed under the following operations:

- nullary operators for falsity $\perp$ and for truth $\top$;

- unary and binary syntactic operations for negation $\neg$, conditionality $\rightarrow$ and conjunction $\wedge$, respectively;

- unary operation for modality $\square_{t}$ with $t \in T$.

Other such operations are defined in terms of those in usual ways. The intended interpretation of $\square_{t} \varphi$ is the sentence that 'trader $t$ knows a sentence $\varphi$.'

A modal logic $L$ is a set of sentences containing all truth-functional tautologies and closed under substitution and modus ponens. A modal logic $L^{\prime}$ is an extension of $L$ if $L \subseteq L^{\prime}$. A sentence $\varphi$ in a modal logic $L$ is a theorem of $L$, written by $\vdash_{L} \varphi$. Other proof-theoretical notions such as $L$-deducibility, L-consistency, L-maximality are defined in usual ways. (See, Chellas [3].)

A system of traders' knowledge is a modal logic $L$ closed under the $2 n+3$ rules of inference $\left(\mathrm{RE}_{\square}\right)$ and containing the schema $(\mathrm{N}),(\mathrm{M}),(\mathrm{C})$, and $(\mathrm{T})$ : For every $t \in T$,

$$
\frac{\varphi \longleftrightarrow \psi}{\square_{t} \varphi \longleftrightarrow \square_{t} \psi}
$$

(N) $\square_{t} \top$;

(M) $\square_{t}(\varphi \wedge \psi) \longrightarrow\left(\square_{t} \varphi \wedge \square_{t} \psi\right)$;

(C) $\quad\left(\square_{t} \varphi \wedge \square_{t} \psi\right) \longrightarrow \square_{t}(\varphi \wedge \psi)$;

(T) $\square_{t} \varphi \longrightarrow \varphi$.

Definition 1. The multi-modal logic KT is the minimal system of trades' knowledge.

\subsection{Information and Knowledge ${ }^{3}$}

Trader $t$ 's information structure is a couple $\left\langle\Omega, P_{t}\right\rangle$, in which $\Omega$ be a non-empty set and $P_{t}$ is a mapping of $\Omega$ into $2^{\Omega}$. It is said to be reflexive if

Ref $\omega \in P_{t}(\omega)$ for every $\omega \in \Omega$,

\footnotetext{
${ }^{3}$ See Fagin, Halpern et al [6].
} 
and it is said to be transitive if

$\operatorname{Trn} \quad \xi \in P_{t}(\omega)$ implies $P_{t}(\xi) \subseteq P_{t}(\omega)$ for any $\xi, \omega \in \Omega$.

An information structure is a structure $\left\langle\Omega,\left(P_{t}\right)_{t \in T}\right\rangle$ where $\Omega$ is common for all trader, and it is called an RT-information structure if each $P_{t}$ is reflexive and transitive.

Given our interpretation, a trader $t$ for whom $P_{t}(\omega) \subseteq E$ knows, in the state $\omega$, that some state in the event $E$ has occurred. In this case we say that at the state $\omega$ the trader $t$ knows $E$. $i$ 's knowledge operator $K_{t}$ on $2^{\Omega}$ is defined by $K_{t}(E)=\left\{\omega \in \Omega \mid P_{t}(\omega) \subseteq E\right\}$. The set $P_{t}(\omega)$ will be interpreted as the set of all the states of nature that $t$ knows to be possible at $\omega$, and $K_{t} E$ will be interpreted as the set of states of nature for which $t$ knows $E$ to be possible. We will therefore call $P_{t}$ t's possibility operator on $\Omega$ and also will call $P_{t}(\omega)$ t's possibility set at $\omega$. A possibility operator $P_{t}$ is determined by the knowledge operator $K_{t}$ such as $P_{t}(\omega)=\bigcap_{K_{t} E \ni \omega} E$. However it is also noted that the operator $P_{t}$ cannot be uniquely determined by the knowledge operator $K_{t}$ when $P_{t}$ does not satisfy the both conditions Ref and Trn.

A partitional information structure is an $R T$-information structure $\left\langle\Omega,\left(P_{t}\right)_{t \in T}\right\rangle$ with the additional condition: For each $t \in T$ and every $\omega \in \Omega$,

Sym $\xi \in P_{t}(\omega)$ implies $P_{t}(\xi) \ni \omega$.

\subsection{Finite Model Property}

A model on an information structure is a triple $\mathcal{M}=\left\langle\Omega,\left(P_{t}\right)_{t \in T}, V\right\rangle$, in which $\left\langle\Omega,\left(P_{t}\right)_{t \in T}\right\rangle$ is an information structure and a mapping $V$ assigns either true or false to every $\omega \in \Omega$ and to every atomic sentence $\mathbf{P}_{m}$. The model $\mathcal{M}$ is called finite if $\Omega$ is a finite set.

Definition 2. By $\models_{\omega}^{\mathcal{M}} \varphi$, we mean that a sentence $\varphi$ is true at a state $\omega$ in a model $\mathcal{M}$. Truth at a state $\omega$ in $\mathcal{M}$ is defined by the inductive way as follows:

1. $\models{ }_{\omega}^{\mathcal{M}} \mathbf{P}_{m}$ if and only if $V\left(\omega, \mathbf{P}_{m}\right)=$ true, for $m=0,1,2, \ldots$;

2. $\models \models_{\omega}^{\mathcal{M}} \top$, and $\operatorname{not} \models \models_{\omega}^{\mathcal{M}} \perp$;

3. $\models_{\omega}^{\mathcal{M}} \neg \varphi$ if and only if not $\models_{\omega}^{\mathcal{M}} \varphi$;

4. $\quad \models_{\omega}^{\mathcal{M}} \varphi \longrightarrow \psi$ if and only if $\models_{\omega}^{\mathcal{M}} \varphi$ implies $\models_{\omega}^{\mathcal{M}} \psi$;

5. $\quad \models_{\omega}^{\mathcal{M}} \varphi \wedge \psi$ if and only if $\models_{\omega}^{\mathcal{M}} \varphi$ and $\models_{\omega}^{\mathcal{M}} \psi$;

6. $\quad \models{ }_{\omega}^{\mathcal{M}} \square_{t} \varphi$ if and only if $P_{t}(\omega) \subseteq\|\varphi\|^{\mathcal{M}}$, for $t \in T$;

Where $\|\varphi\|^{\mathcal{M}}$ denotes the set of all the states in $\mathcal{M}$ at which $\varphi$ is true; this is called the truth set of $\varphi$. We say that a sentence $\varphi$ is true in the model $\mathcal{M}$ and write $\models{ }^{\mathcal{M}} \varphi$ if $=_{\omega}^{\mathcal{M}} \varphi$ for every state $\omega$ in $\mathcal{M}$. A sentence is said to be valid in an information structure if it is true in every model on the information structure. 
Let $\Sigma$ be a set of sentences. We say that $\mathcal{M}$ is a model for $\Sigma$ if every member of $\Sigma$ is true in $\mathcal{M}$. An information structure is said to be for $\Sigma$ if every member of $\Sigma$ is valid in it. Let $\boldsymbol{R}$ be a class of models on a reflexive information structure. A modal logic $L$ is sound with respect to $\boldsymbol{R}$ if every member of $\boldsymbol{R}$ is a model for $L$. It is complete with respect to $\boldsymbol{R}$ if every sentence valid in all members of $\boldsymbol{R}$ is a theorem of $L$. We say that $L$ is determined by $\boldsymbol{R}$ if $L$ is sound and complete with respect to $\boldsymbol{R}$.

A modal logic $L$ is said to have the finite model property if it is determined by the class of all finite models in $\boldsymbol{R}$. The following theorem can be shown by the same way described in Chellas [3].

Theorem 1. The multi-modal logic KT has the finite model property.

From now on we consider $t$ 's information structure $\left\langle\Omega,\left(P_{t}\right)_{t \in T}, V\right\rangle$ as a finite model for $\mathbf{K} \mathbf{T}_{t}$.

\subsection{Economy for Logic KT}

Let $\Omega$ be a non-empty finite set called a state space, and let $2^{\Omega}$ denote the field of all subsets of $\Omega$. Each member of $2^{\Omega}$ is called an event and each element of $\Omega$ a state. The space of the traders is a measurable space $(T, \Sigma, \mu)$ in which $T$ is a set of traders, $\Sigma$ is a $\sigma$-field of subsets of $T$ whose elements are called coalitions, and $\mu$ is a measure on $\Sigma$.

A pure exchange economy under uncertainty is a tuple $\left\langle T, \Sigma, \mu, \Omega, e,\left(U_{t}\right)_{t \in T}\right.$, $\left.\left(\pi_{t}\right)_{t \in T}\right\rangle$ consisting of the following structure and interpretations: There are $l$ commodities in each state of the state space $\Omega$, and it is assumed that $\Omega$ is finite and that the consumption set of trader $t$ is $\mathbb{R}_{+}^{l}$;

- $(T, \Sigma, \mu)$ is the measure space of the traders;

- $\boldsymbol{e}: T \times \Omega \rightarrow \mathbb{R}_{+}^{l}$ is $t$ 's initial endowment such that $\boldsymbol{e}(\cdot, \omega)$ is $\mu$-measurable for each $\omega \in \Omega$;

$-U_{t}: \mathbb{R}_{+}^{l} \times \Omega \rightarrow \mathbb{R}$ is $t$ 's von-Neumann and Morgenstern utility function;

- $\pi_{t}$ is a subjective prior on $\Omega$ for a trader $t \in T$.

For simplicity it is assumed that $\left(\Omega, \pi_{t}\right)$ is a finite probability space with $\pi_{t}$ full support ${ }^{4}$ for almost all $t \in T$.

Definition 3. An pure exchange economy for logic $\mathbf{K T}$ is a structure $\mathcal{E}^{K T}=$ $\left\langle\mathcal{E},\left(P_{t}\right)_{t \in T}, V\right\rangle$, in which $\mathcal{E}$ is a pure exchange economy such that $\left\langle\Omega,\left(P_{t}\right)_{t \in T}, V\right\rangle$ is a finite model for the logic KT. Furthermore it is called an economy under $R T$-information structure if each $P_{t}$ is a reflexive and transitive information structure.

Remark 1. An economy under asymmetric information is an economy $\mathcal{E}^{K T}$ under partitional information structure (i.e., each $P_{t}$ satisfies the three conditions Ref, Trn and Sym.)

\footnotetext{
${ }^{4}$ I.e., $\pi_{t}(\omega) \supsetneqq 0$ for every $\omega \in \Omega$.
} 
Let $\mathcal{E}^{K T}$ be a pure exchange economy for logic $\mathbf{K T}$. We denote by $\mathcal{F}_{t}$ the field generated by $\left\{P_{t}(\omega) \mid \omega \in \Omega\right\}$ and by $\mathcal{F}$ the join of all $\mathcal{F}_{t}(t \in T)$; i.e. $\mathcal{F}=\vee_{t \in T} \mathcal{F}_{t}$. We denote by $\{A(\omega) \mid \omega \in \Omega\}$ the set of all atoms $A(\omega)$ containing $\omega$ of the field $\mathcal{F}=\vee_{t \in T} \mathcal{F}_{t}$.

Remark 2. The set of atoms $\left\{A_{t}(\omega) \mid \omega \in \Omega\right\}$ of $\mathcal{F}_{t}$ does not necessarily coincide with the partition induced from $P_{t}$.

We shall often refer to the following conditions: For every $t \in T$,

A-1 $\quad \int_{T} \boldsymbol{e}(t, \omega) d \mu \supsetneqq 0$ for all $\omega \in \Omega$.

A-2 $\boldsymbol{e}(t, \cdot)$ is $\mathcal{F}_{t}$-measurable

A-3 For each $x \in \mathbb{R}_{+}^{l}$, the function $U_{t}(x, \cdot)$ is $\mathcal{F}_{t}$-measurable, and the function: $T \times \mathbb{R}_{+}^{l} \rightarrow \mathbb{R},(t, x) \mapsto U_{t}(x, \omega)$ is $\Sigma \times \mathcal{B}$-measurable where $\mathcal{B}$ is the $\sigma$-field of all Borel subsets of $\mathbb{R}_{+}^{l}$.

A-4 For each $\omega \in \Omega$, the function $U_{t}(\cdot, \omega)$ is continuous, strictly increasing on $\mathbb{R}_{+}^{l}$.

A-5 For each $\omega \in \Omega$, the function $U_{t}(\cdot, \omega)$ is continuous, increasing, strictly quasi-concave and non-saturated ${ }^{5}$ on $\mathbb{R}_{+}^{l}$.

Remark 3. It is plainly observed that A-5 implies $\mathbf{A - 4}$. We note also that $\mathbf{A - 3}$ does not mean that trader $t$ knows his/her utility function $U_{t}(\cdot, \omega){ }^{6}$

\subsection{Ex-post Core}

An assignment $\boldsymbol{x}$ is a mapping from $T \times \Omega$ into $\mathbb{R}_{+}^{l}$ such that for every $\omega \in \Omega$, the function $\boldsymbol{x}(\cdot, \omega)$ is $\mu$-measurable, and for each $t \in T$, the function $\boldsymbol{x}(t, \cdot)$ is at most $\mathcal{F}$-measurable. We denote by $\mathcal{A s s}\left(\mathcal{E}^{K T}\right)$ the set of all assignments for the economy $\mathcal{E}^{K T}$.

By an allocation we mean an assignment $\boldsymbol{a}$ such that for every $\omega \in \Omega$,

$$
\int_{T} \boldsymbol{a}(t, \omega) d \mu \leqq \int_{T} \boldsymbol{e}(t, \omega) d \mu .
$$

We denote by $\mathcal{A} l c\left(\mathcal{E}^{K T}\right)$ the set of all allocations, and for each $t \in T$ we denote by $\mathcal{A} l c\left(\mathcal{E}^{K T}\right)_{t}$ the set of all the functions $\boldsymbol{a}(t, \cdot)$ for $\boldsymbol{a} \in \mathcal{A l c}\left(\mathcal{E}^{K T}\right)$.

An assignment $\boldsymbol{y}$ is called an ex-post improvement of a coalition $S \in \Sigma$ on an assignment $\boldsymbol{x}$ at a state $\omega \in \Omega$ if

$\operatorname{Imp1} \mu(S) \supsetneqq 0$;

Imp2 $\int_{S} \boldsymbol{y}(t, \omega) d \mu \leqq \int_{S} \boldsymbol{e}(t, \omega) d \mu ; \quad$ and

Imp3 $U_{t}(\boldsymbol{y}(t, \omega), \omega) \supsetneqq U_{t}(\boldsymbol{x}(t, \omega), \omega)$ for almost all $t \in S$.

We shall present the notion of core in an economy $\mathcal{E}^{K T}$ for logic $\mathbf{K T}$.

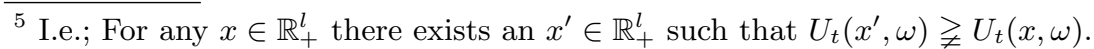

${ }^{6}$ That is, $\omega \notin K_{t}\left(\left[U_{t}(\cdot, \omega)\right]\right)$ for some $\omega \in \Omega$, where $\left[U_{t}(\cdot, \omega)\right]:=\left\{\xi \in \Omega \mid U_{t}(\cdot, \xi)=\right.$ $\left.U_{t}(\cdot, \omega)\right\}$. This is because the information structure is not a partitional structure.
} 
Definition 4. An allocation $\boldsymbol{x}$ is said to be an ex-post core allocation of a pure exchange economy for logic $\mathbf{K T}$ if there is no coalition having an ex-post improvement on $\boldsymbol{x}$ at any state $\omega \in \Omega$. The ex-post core denoted by $\mathcal{C}^{E x P}\left(\mathcal{E}^{K T}\right)$ is the set of all the ex-post core allocations of $\mathcal{E}^{K T}$.

Let $\mathcal{E}^{K T}$ be the pure exchange economy for logic $\mathbf{K T}$ and $\mathcal{E}^{K T}(\omega)$ the economy with complete information $\left\langle T, \Sigma, \mu, \boldsymbol{e}(\cdot, \omega),\left(U_{t}(\cdot, \omega)\right)_{t \in T}\right\rangle$ for each $\omega \in \Omega$. We denote by $C\left(\mathcal{E}^{K T}(\omega)\right)$ the set of all core allocations for $\mathcal{E}^{K T}(\omega)$.

Proposition 1. Let $\mathcal{E}^{K T}$ be a pure exchange economy for logic $\mathbf{K T}$ satisfying the conditions $\mathbf{A - 1}, \mathbf{A - 2}$ and $\mathbf{A - 3}$. Suppose that the economy is atomless (that is, $(T, \Sigma, \mu)$ is non-atomic measurable space.) The ex-post core of $\mathcal{E}^{K T}$ is nonempty (i.e., $\left.\mathcal{C}^{\operatorname{ExP}}\left(\mathcal{E}^{K T}\right) \neq \emptyset\right)$. Moreover, $\mathcal{C}^{E x P}\left(\mathcal{E}^{K T}\right)$ coincides with the set of all assignments $\boldsymbol{x}$ such that $\boldsymbol{x}(\cdot, \omega)$ is a core allocation for the economy $\mathcal{E}^{K T}(\omega)$ for all $\omega \in \Omega$ : i.e.,

$$
\left.\mathcal{C}^{E x P}\left(\mathcal{E}^{K T}\right)=\left\{\boldsymbol{x} \in \mathcal{A} l c\left(\mathcal{E}^{K T}\right) \mid \boldsymbol{x}(\cdot, \omega)\right) \in \mathcal{C}\left(\mathcal{E}^{K T}(\omega)\right) \text { for all } \omega \in \Omega\right\} .
$$

\subsection{Expectation and Pareto Optimality}

Let $\mathcal{E}^{K T}$ be the pure exchange economy for logic $\mathbf{K T}$. We denote by $\mathbf{E}_{t}\left[U_{t}(\boldsymbol{x}(t, \cdot)]\right.$ the ex-ante expectation defined by

$$
\mathbf{E}_{t}\left[U_{t}(\boldsymbol{x}(t, \cdot)]:=\sum_{\omega \in \Omega} U_{t}(\boldsymbol{x}(t, \omega), \omega) \pi_{t}(\omega)\right.
$$

for each $\boldsymbol{x} \in \mathcal{A} s s\left(\mathcal{E}^{K T}\right)$. We denote by $\mathbf{E}_{t}\left[U_{t}(\boldsymbol{x}(t, \cdot)) \mid P_{t}\right](\omega)$ the interim expectation defined by

$$
\mathbf{E}_{t}\left[U_{t}\left(\boldsymbol{x}(t, \cdot) \mid P_{t}\right](\omega):=\sum_{\xi \in \Omega} U_{t}(\boldsymbol{x}(t, \xi), \xi) \pi_{t}\left(\xi \mid P_{t}(\omega)\right) .\right.
$$

Definition 5. An allocation $\boldsymbol{x}$ in an economy $\mathcal{E}^{K T}$ is said to be ex-ante Paretooptimal if there is no allocation $\boldsymbol{a}$ with the two properties as follows:

PO-1 For almost all $t \in T, \mathbf{E}_{t}\left[U_{t}(\boldsymbol{a}(t, \cdot)] \geqq \mathbf{E}_{t}\left[U_{t}(\boldsymbol{x}(t, \cdot)]\right.\right.$.

PO-2 The set of all the traders $s \in T$ such that

$$
\mathbf{E}_{s}\left[U _ { s } ( \boldsymbol { a } ( t , \cdot ) ] \supsetneqq \mathbf { E } _ { s } \left[U_{s}(\boldsymbol{x}(t, \cdot)]\right.\right.
$$

is not a $\mu$-null set.

\subsection{Rational Expectations Equilibrium}

Let $\mathcal{E}^{K T}=\left\langle N, \Omega,\left(e_{t}\right)_{t \in T},\left(U_{t}\right)_{t \in T},\left(\pi_{t}\right)_{t \in T},\left(P_{t}\right)_{t \in T}\right\rangle$ be a pure exchange economy for logic KT. A price system is a non-zero $\mathcal{F}$-measurable function $p: \Omega \rightarrow$ $\mathbb{R}_{+}^{l}$. We denote by $\sigma(p)$ the smallest $\sigma$-field that $p$ is measurable, and by $\Delta(p)(\omega)$ 
the atom containing $\omega$ of the field $\sigma(p)$. The budget set of a trader $t$ at a state $\omega$ for a price system $p$ is defined by

$$
B_{t}(\omega, p):=\left\{x \in \mathbb{R}_{+}^{l} \mid p(\omega) \cdot x \leqq p(\omega) \cdot e(t, \omega)\right\} .
$$

Let $\Delta(p) \cap P_{t}: \Omega \rightarrow 2^{\Omega}$ be defined by $\left(\Delta(p) \cap P_{t}\right)(\omega):=\Delta(p)(\omega) \cap P_{t}(\omega)$; it is plainly observed that the mapping $\Delta(p) \cap P_{t}$ satisfies Ref. We denote by $\sigma(p) \vee \mathcal{F}_{t}$ the smallest $\sigma$-field containing both the fields $\sigma(p)$ and $\mathcal{F}_{t}$, and by $A_{t}(p)(\omega)$ the atom containing $\omega$. It is noted that

$$
A_{t}(p)(\omega)=\left(\Delta(p) \cap A_{t}\right)(\omega) .
$$

Remark 4. If $P_{t}$ satisfies Ref and Trn then $\sigma(p) \vee \mathcal{F}_{t}$ coincides with the field generated by $\Delta(p) \cap P_{t}$.

We shall propose the extended notion of rational expectations equilibrium for an economy $\mathcal{E}^{K T}$.

Definition 6. A rational expectations equilibrium for an economy $\mathcal{E}^{K T}$ under reflexive information structure is a pair $(p, \boldsymbol{x})$, in which $p$ is a price system and $\boldsymbol{x}$ is an allocation satisfying the following conditions:

RE 1 For almost all $t \in T, \boldsymbol{x}(t, \cdot)$ is $\sigma(p) \vee \mathcal{F}_{t}$-measurable.

RE 2 For almost all $t \in T$ and for every $\omega \in \Omega, \boldsymbol{x}(t, \omega) \in B_{t}(\omega, p)$.

RE 3 For almost all $t \in T$, if $\boldsymbol{y}(t, \cdot): \Omega \rightarrow \mathbb{R}_{+}^{l}$ is $\sigma(p) \vee \mathcal{F}_{t}$-measurable with $\boldsymbol{y}(t, \omega) \in B_{t}(\omega, p)$ for all $\omega \in \Omega$, then

$$
\mathbf{E}_{t}\left[U_{t}(\boldsymbol{x}(t, \cdot)) \mid \Delta(p) \cap P_{t}\right](\omega) \geqq \mathbf{E}_{t}\left[U_{t}(\boldsymbol{y}(t, \cdot)) \mid \Delta(p) \cap P_{t}\right](\omega)
$$

pointwise on $\Omega$.

RE 4 For every $\omega \in \Omega, \int_{T} \boldsymbol{x}(t, \omega) d \mu=\int_{T} \boldsymbol{e}(t, \omega) d \mu$.

The allocation $\boldsymbol{x}$ in $\mathcal{E}^{K T}$ is called a rational expectations equilibrium allocation.

We denote by $R E\left(\mathcal{E}^{K T}\right)$ the set of all the rational expectations equilibria of a pure exchange economy $\mathcal{E}^{K T}$ for logic $\mathbf{K T}$, and denote by $\mathcal{R}\left(\mathcal{E}^{K T}\right)$ the set of all the rational expectations equilibrium allocations for the economy

\section{Existence Theorem}

We can prove the existence theorem of the generalized rational expectations equilibrium for a pure exchange economy $\mathcal{E}^{K T}$ for logic KT. Let $\mathcal{E}^{K T}(\omega)$ be the economy with complete information for each $\omega \in \Omega$. We set by $W\left(\mathcal{E}^{K T}(\omega)\right)$ the set of all the competitive equilibria for $\mathcal{E}^{K T}(\omega)$, and we denote by $\mathcal{W}\left(\mathcal{E}^{K T}(\omega)\right)$ the set of all the competitive equilibrium allocations for $\mathcal{E}^{K T}(\omega)$.

Theorem 2. Let $\mathcal{E}^{K T}$ be a pure exchange economy for logic $\mathbf{K T}$ satisfying the conditions A-1, A-2, A-3 and A-4. Suppose that the economy is atomless (that is, $(T, \Sigma, \mu)$ is non-atomic measurable space.) Then there exists a rational expectations equilibrium for the economy; i.e., $R E\left(\mathcal{E}^{K T}\right) \neq \emptyset$.

Proof. See Appendix.

Remark 5. Matsuhisa and Ishikawa [8] shows Theorem 2 for an economy under $R T$-information structure. 


\section{Proof of Main Theorem}

We can now state explicitly Main theorem in Section 1 as follows:

Theorem 3. Let $\mathcal{E}^{K T}$ be a pure exchange economy for logic KT satisfying the conditions $\mathbf{A - 1}, \mathbf{A - 2}, \mathbf{A - 3}$ and $\mathbf{A - 4}$. Suppose that the economy is atomless (that is, $(T, \Sigma, \mu)$ is non-atomic measurable space.) Then the ex-post core coincides with the set of all rational expectations equilibrium allocations; i.e., $\mathcal{C}^{E x P}\left(\mathcal{E}^{K T}\right)=\mathcal{R}\left(\mathcal{E}^{K T}\right)$.

In view of Theorem 2 it is first noted that $\mathcal{R}\left(\mathcal{E}^{K T}\right) \neq \emptyset$. Because $\mathcal{E}^{K T}(\omega)$ is an atomless pure exchange economy for each $\omega \in \Omega$, it follows from the core equivalence theorem of Aumann [1] that $\mathcal{C}\left(\mathcal{E}^{K T}(\omega)\right)=\mathcal{W}\left(\mathcal{E}^{K T}(\omega)\right)$ for any $\omega \in \Omega$. We shall observe that Main theorem immediately follows from the above Proposition 1 together with the below Proposition 2:

Proposition 2. Let $\mathcal{E}^{K T}$ be a pure exchange economy for logic $\mathbf{K T}$ satisfying the conditions A-1, A-2, A-3 and $\mathbf{A - 4}$. Then the set of all rational expectations equilibrium allocations $\mathcal{R}\left(\mathcal{E}^{K T}\right)$ coincides with the set of all the assignments $\boldsymbol{x}$ such that $\boldsymbol{x}(\cdot, \omega)$ is a competitive equilibrium allocation for the economy with complete information $\mathcal{E}^{K T}(\omega)$ for all $\omega \in \Omega$; i.e.,

$$
\begin{aligned}
\mathcal{R}\left(\mathcal{E}^{K T}\right)=\left\{\boldsymbol{x} \in \mathcal{A l c}\left(\mathcal{E}^{K T}\right) \mid\right. & \text { There is a price system } p \text { such that } \\
& \left.(p(\omega), \boldsymbol{x}(\cdot, \omega)) \in W\left(\mathcal{E}^{K T}(\omega)\right) \text { for all } \omega \in \Omega\right\} .
\end{aligned}
$$

\section{Concluding Remarks}

We shall give a remark about the ancillary assumptions in results in this article. Could we prove the theorems under the generalized information structure removing out the reflexivity? The answer is no vein. If trader $t$ 's possibility operator does not satisfy Ref then his/her expectation with respect to a price cannot be defined at a state because it is possible that $\Delta(p)(\omega) \cap P_{t}(\omega)=\emptyset$ for some $\omega \in \Omega$.

Could we prove the theorems without four conditions A-1, A-2, A-3 and A-4 together with A-5. The answer is no again. The suppression of any of these assumptions renders the existence theorem of rational expectations equilibrium (Theorem 2) vulnerable to the discussion and the example proposed in Remarks 4.6 of Matsuhisa and Ishikawa (2002).

\section{Appendix}

\section{Proof of Theorem 2}

In view of the conditions $\mathbf{A - 1}, \mathbf{A - 2}, \mathbf{A - 3}$ and $\mathbf{A - 4}$, it follows from the existence theorem of a competitive equilibrium for an atomless economy with com- 
plete information ${ }^{7}$ that for each $\omega \in \Omega$, there exists a competitive equilibrium $\left(p^{*}(\omega), \boldsymbol{x}^{*}(\cdot, \omega)\right) \in W\left(\mathcal{E}^{K T}(\omega)\right)$. We take a set of strictly positive numbers $\left\{k_{\omega}\right\}_{\omega \in \Omega}$ such that $k_{\omega} p^{*}(\omega) \neq k_{\xi} p^{*}(\xi)$ for any $\omega \neq \xi$. We define the pair $(p, \boldsymbol{x})$ as follows: For each $\omega \in \Omega$ and for all $\xi \in A(\omega), p(\xi):=k_{\omega} p^{*}(\omega)$ and $\boldsymbol{x}(t, \xi):=$ $\boldsymbol{x}^{*}(t, \omega)$. It is noted that $\boldsymbol{x}(\cdot, \xi) \in W\left(\mathcal{E}^{K T}(\omega)\right)$ because $\mathcal{E}^{K T}(\xi)=\mathcal{E}^{K T}(\omega)$, and we note that $\Delta(p)(\omega)=A(\omega)$.

We shall verify that $(p, \boldsymbol{x})$ is a rational expectations equilibrium for $\mathcal{E}^{K T}$ : In fact, it is easily seen that $p$ is $\mathcal{F}$-measurable with $\Delta(p)(\omega)=A(\omega)$ and that $\boldsymbol{x}(t, \cdot)$ is $\sigma(p) \vee \mathcal{F}_{t}$-measurable, so RE 1 is valid. Because $\left(\Delta(p) \cap P_{t}\right)(\omega)=A(\omega)$ for every $\omega \in \Omega$, it can be plainly observed that $\boldsymbol{x}(t, \cdot)$ satisfies $\mathbf{R E} \mathbf{2}$, and it follows from A-3 that for almost all $t \in T, \mathbf{E}_{t}\left[U_{t}(\boldsymbol{x}(t, \cdot)) \mid \Delta(p) \cap P_{t}\right](\omega)=U_{t}(\boldsymbol{x}(t, \omega), \omega)$ On noting that $\mathcal{E}^{K T}(\xi)=\mathcal{E}^{K T}(\omega)$ for any $\xi \in A(\omega)$, it is plainly observed that $(p(\omega), \boldsymbol{x}(t, \omega))=\left(k_{\omega} p^{*}(\omega), \boldsymbol{x}^{*}(t, \omega)\right)$ is also a competitive equilibrium for $\mathcal{E}^{K T}(\omega)$ for every $\omega \in \Omega$, and it can be observed by the above equation that RE $\mathbf{3}$ is valid for $(p, \boldsymbol{x})$, in completing the proof.

\section{References}

1. Aumann, R. J.: Markets with a continuum of traders, Econometrica 32 (1964) 39-50.

2. Bacharach, M. O.: Some extensions of a claim of Aumann in an axiomatic model of knowledge, Journal of Economic Theory 37 (1985) 167-190.

3. Chellas, B. F.: Modal Logic: An introduction. Cambridge University Press, Cambridge, London, New York, New Rochelle, Melbourne, Sydney (1980)

4. Debreu, G.: Existence of competitive equilibrium. In Arrow, K.J. and Intriligator, M.D., (eds): Handbook of Mathematical Economics, Volume 2. North-Holland Publishing Company, Amsterdam (1982) 697-744.

5. Einy, E., Moreno, D. and Shitovitz, B.: Rational expectations equilibria and the ex-post core of an economy with asymmetric information, Journal of Mathematical Economics 34 (2000) 527-535.

6. Fagin, R., Halpern, J.Y., Moses, Y., and Vardi, M.Y. Reasoning about Knowledge. The MIT Press, Cambridge, Massachusetts, London, England (1995)

7. Geanakoplos, J.: Game theory without partitions, and applications to speculation and consensus, Cowles Foundation Discussion Paper No.914 (1989) (Available in http://cowles.econ.yale.edu)

8. Matsuhisa, T. and Ishikawa, R.: Rational expectations can preclude trades. Working paper. Hitotsubashi Discussion Paper Series No.2002-1 (2002) (Available in http://wakame.econ.hit-u.ac.jp/)

9. Matsuhisa, T., Ishikawa, R. and Hoshino, Y.: Core equivalence in economy under generalized information. Working paper. Hitotsubashi Discussion Paper Series No.2002-12 (2002) (Available in http://wakame.econ.hit-u.ac.jp/)

10. Volij, O.,: Communication, credible improvements and the core of an economy with asymmetric information, International Journal of Game Theory 29 (2000) 63-79.

11. Wilson, R.: Information, efficiency, and the core of an economy, Econometrica 40 (1978) 807-816.

$\overline{7}$ C.f., Theorem 9 in Debreu [4] 